\title{
A Generalized 4D Image Registration Scheme for Targeted Radionuclide Therapy Dosimetry
}

\author{
Periklis Papavasileiou, ${ }^{1}$ Antigoni Divoli, ${ }^{2}$ Kostas Hatziioannou, ${ }^{1}$ and Glenn D. Flux ${ }^{2}$ \\ ${ }^{1}$ Department of Medical Physics, Papageorgiou General Hospital, Thessaloniki, Greece \\ ${ }^{2}$ Joint Department of Physics, Institute of Cancer Research and Royal Marsden Hospital NHS \\ Foundation Trust, Sutton, Surrey, United Kingdom
}

\begin{abstract}
An iterative, generalized four-dimensional (4D) method is presented in this paper that allows simultaneous registration of a series of single-photon emission computed tomography (SPECT) scans acquired in the course of a radionuclide therapy or pretherapy tracer study. The method combines temporal information with voxel-based similarity criteria to carry out simultaneous registration of the SPECT scans. A polynomial function was fitted to the maximum counts of each tumor site over the 4D study. Each tumor site was normalized to its maximum on the reference scan, and a template $4 D$ dataset was generated, employing the polynomial fitting and the normalization map. Then, each $3 D$ scan was registered to the corresponding simulated scan, using a $3 D$ similarity criterion. The correlation coefficient $(C C)$, the mutual information (MI), and the sum-of-absolute differences (SAD) similarity criteria were employed. Simulated data, based on a head-neck ${ }^{131} I-M I B G$ study, were used to compare the proposed method for $4 D$ registration with sequential $3 D$ registration. Sequential $3 D$ registration resulted in residual registration errors of $3.5 \pm 2.5,3.2 \pm 2.0$, and $7.0 \pm 3.5 \mathrm{~mm}$ for the CC, MI, and SAD criteria respectively, whereas the corresponding $4 D$ method gave errors of $2.4 \pm 1.6,1.9 \pm 1.1$, and $5.3 \pm 2.9 \mathrm{~mm}$ for the CC, MI, and SAD criteria, respectively. The $4 D$ method was applied to ${ }^{186}$ Re HEDP SPECT patient studies and registration was verified by a dual-cursor display tool.
\end{abstract}

Key words: image registration, radionuclide therapy dosimetry, polynomial fitting

\section{INTRODUCTION}

In three-dimensional (3D) dosimetry for targeted radionuclide therapy (TRT), the administration of a radionuclide-labeled, tumor-specific agent is followed by a series of sequential (SPECT) single-photon emission computed tomography scans

Address reprint requests to: Periklis Papavasileiou; Department of Medical Physics, Papageorgiou General Hospital; Nea Efkarpia's Ring Road, 54603, Thessaloniki, Greece; Tel.: +0030-2310-693420

E-mail: Ppapavasileiou@yahoo.gr (i.e., a 4D dataset) to characterize the absorbeddose distribution to tumor sites. ${ }^{1-4}$ Registration of these scans is essential to allow generation of 3D absorbed dose distributions.

Manual registration was employed by Kolbert et al. ${ }^{2,3}$ in radiolabeled antibody therapy. Markerbased registration has also been employed. ${ }^{5,6}$ Voxel-based registration methods ${ }^{7}$ rely on the image information to define similarity criteria, optimized at registration. The similarity criteria used in 3D voxel-based methods attempt registration of just two scans; in order to register a 4D study, each scan of the series is registered to its immediate predecessor (sequential voxel-based 
registration) or a scan is named as the reference scan for all remaining scans to be registered to.

In contrast to sequential voxel-based registration methods, 4D methods compute the similarity criterion by taking into account both spatial and temporal information. A 4D method for registration was described ${ }^{5}$ to register a series of SPECT scans, assuming that a monoexponential decay function could be fitted to the data. In this paper, a generalized, iterative, 4D registration method for TRT is presented, which combined temporal information with voxel-based 3D similarity criteria (spatial information). The 4D method was compared with 3D sequential registration, employing three similarity criteria: the mutual information (MI), the correlation coefficient (CC), and the sum of absolute differences (SAD). The 4D method was also applied to ${ }^{186} \mathrm{Re}$ HEDP patient data. The registration and image analysis software was written in MATLAB, version 6.5.1 (MathWorks, Inc., Natick, MA).

\section{MATERIALS AND METHODS}

\section{The 4D Registration Method}

The developed, iterative 4D registration algorithm consists of the following steps:

1. The $4 \mathrm{D}$ dataset is defined as $\{\mathrm{DS}\}=\left\{\mathrm{DS}_{1}\right.$, $\left.\mathrm{DS}_{2}, \ldots, \mathrm{DS}_{N}\right\}$, where $\mathrm{DS}_{i}$ corresponds to the $i$ th scan and $N$ is the number of scans.

2. Individual volumes of interest (VOIs) are defined around each tumor site and/or normal organ on each scan $\mathrm{DS}_{i}$; the user draws the VOIs employing an image analysis tool, developed for that purpose. No thresholding is employed on the user-defined VOIs. The reference scan can be any of the individual SPECT scans.

3. The maximum counts of the VOI on each scan $\mathrm{DS}_{i}$ are computed and fitted by a $m$ th order polynomial, where $1 \leq m<N$.

4. The tumor VOI is normalized to its maximum value, on the reference scan, and a 3D normalization map is generated.

5. A template study, $\left\{\mathrm{DS}_{\mathrm{S}}\right\}$, based on the polynomial coefficients and the normalization map, is generated. The assumption is made that the spatial activity distribution within the tumor site remains constant throughout the 4D study.

6. Two temporary datasets, $\left\{\mathrm{DS}_{\mathrm{I}}\right\}$ and $\left\{\mathrm{DS}_{\mathrm{T}}\right\}$, are created. $\left\{\mathrm{DS}_{\mathrm{I}}\right\}$ consists of the individual scans prior to an iteration of the $4 \mathrm{D}$ algorithm and is initially set equal to $\{D S\}$. $\left\{D^{T}\right\}$ is updated every time an individual scan is transformed during an iteration of the 4D algorithm, and is initially set equal to $\left\{\mathrm{DS}_{\mathrm{S}}\right\}$.

7. The number of iterations, $k$, is set to 1 .

8. The scan index $i$ is set to 2 , assuming the first scan is the reference. For each scan, that is, for all values of $i$ between 2 and $N$, steps 9, $10,11,12$, and 13 are performed.

9. The transformation, $\mathrm{T}_{i}^{k}$, to register the $i$ th scan, $\mathrm{DS}_{\mathrm{I}, i}$, to $\mathrm{DS}_{\mathrm{T}, i}$ is determined by use of a 3D similarity criterion, either the MI, the $\mathrm{CC}$, or the SAD. The transformation $\mathrm{T}_{i}^{k}$ incorporates both spatial and image intensity information (i.e., the interpolation method employed to map both position and image intensity; trilinear interpolation was employed to transform the scans).

10. The registered scan, $\mathrm{DS}_{\mathrm{I}, i}{ }^{k}$, is then generated by $\mathrm{DS}_{\mathrm{I}, i}{ }^{k}=\mathrm{T}_{i}^{k} D S_{I, \mathrm{i}}$.

11. $\left\{\mathrm{DS}_{\mathrm{T}}\right\}$ and the transformed $\mathrm{DS}_{\mathrm{I}, i}{ }^{k}$, scan are combined to form an updated dataset, $\left\{\mathrm{DS}_{\mathrm{T}}\right\}$ defined as $\left\{\mathrm{DS}_{\mathrm{T}}\right\}=\left\{\mathrm{DS}_{\mathrm{T}}\right\} \cup \mathrm{DS}_{\mathrm{I}, i}{ }^{k}$, where " $\cup$ " is the symbol of union in set theory.

12. Each voxel $4 \mathrm{D}$ series of the $\left\{\mathrm{DS}_{\mathrm{T}}\right\}$ is fitted by an $m$ th order polynomial, and the polynomial coefficients are saved.

13. The $\left\{\mathrm{DS}_{\mathrm{T}, i+1}{ }^{k}, \ldots, \mathrm{DS}_{\mathrm{T}, N}{ }^{k}\right\}$ are generated, based on the updated polynomial coefficients and the normalization map.

14. The final, registered dataset, $\left\{\mathrm{DS}_{\mathrm{F}}\right\}$, is set equal to $\left\{\mathrm{DS}_{\mathrm{T}}\right\}$.

Following each iteration, the average 3D movement, $d_{i}^{k}$, of each scan is computed, using the following:

$$
d_{i}^{k}=\Sigma\left|\mathrm{T}_{i}^{k}\right| \mathrm{P}
$$

where $\mathrm{T}_{i}{ }^{k}$ is the spatial transformation for the $i t h$ scan in the kth iteration, and $\mathbf{P}$ is the voxel coordinates' vector; the summation in Equation 1 is over all the M voxels of the ith scan's registered volume. If after the $k t h$ iteration there is a $d_{i}^{k}$ above the smallest voxel dimension of the scans, a further iteration is carried out. Otherwise, the registration process is terminated. For the registration processes carried out as presented in this paper, the number of iterations was 2 .

\section{Patient data}

SPECT data from six therapies (Table 1) were used in this paper: $1{ }^{131} \mathrm{I}-\mathrm{MIBG}$ patient (subject 


\begin{tabular}{|c|c|c|c|c|c|c|c|c|}
\hline \multirow[b]{2}{*}{ Patient study } & \multicolumn{8}{|c|}{$\begin{array}{c}\text { Hours following administration of therapeutic amount of } \\
\text { radioctivity }\end{array}$} \\
\hline & 1 & 5 & 24 & 48 & 72 & 96 & 120 & 144 \\
\hline $\mathrm{A}\left({ }^{131} \mathrm{I}-\mathrm{mIBG}\right)$ & & & & - & & & - & - \\
\hline B ( $\left.{ }^{186} \mathrm{Re}-\mathrm{HEDP}\right)$ & - & - & - & - & - & & & \\
\hline C ( $\left({ }^{186} \mathrm{Re}-\mathrm{HEDP}\right)$ & - & - & - & - & & & & \\
\hline $\mathrm{D}\left({ }^{186} \mathrm{Re}-\mathrm{HEDP}\right)$ & - & - & - & - & - & & & \\
\hline E ( $\left.{ }^{186} \mathrm{Re}-\mathrm{HEDP}\right)$ & - & & - & - & - & & & \\
\hline F ( ${ }^{186}$ Re-HEDP) & - & - & - & - & - & & & \\
\hline
\end{tabular}

A) and $5{ }^{186}$ Re-HEDP patients (subjects B, C, D, $\mathrm{E}$, and F). The ${ }^{131} \mathrm{I}$ scans were acquired on a VGMillennium dual-head (5/8 inch crystal) gamma camera (General Electric, Atlanta, GA), with a high-energy, high-resolution collimator (72 views, matrix size $128 \times 128$, pixel dimensions, $4.7 \times$ $4.7 \mathrm{~mm}$ ), employing a triple-energy window (TEW) protocol (primary window, $364 \% \pm 10 \%$; lower scatter window, $317 \% \pm 3 \%$; and an upper scatter window, $415 \% \pm 3 \%$ ). Transaxial images (Fig. 1A) were reconstructed by filtered back projection (FBP), using a Ramp-Hanning filter, with a cutoff frequency of 0.8 of the Nyquist frequency into a 3D matrix (voxel dimensions, $4.7 \times 4.7 \times$ $4.7 \mathrm{~mm}$ ). Attenuation correction was carried out using a first-order Chang correction. ${ }^{8}$

The ${ }^{186}$ Re-HEDP scans were performed with the FORTE HP (ADAC) scanner. A low-energy, general-purpose collimator was used, providing paral- lel beam geometry (64 views, matrix size $128 \times$ 128, pixel dimensions, $4.7 \times 4.7 \mathrm{~mm}$ ), employing a TEW protocol (primary window, $137 \% \pm 15 \%$; lower scatter window, $119 \% \pm 7 \%$; upper scatter window, $156 \% \pm 6 \%$ ). Transaxial images (Fig. 1B) were reconstructed using OSEM (8 iterations and 8 subsets) into a 3D matrix (voxel dimensions, $4.7 \times 4.7 \times 4.7 \mathrm{~mm})$.

\section{Simulated Data}

A 4D simulated dataset was generated, based on the patient $\mathrm{A}{ }^{131} \mathrm{I}-\mathrm{MIBG}$, employing a monoexponential decay function. The simulated dataset, hereafter referred to as $A_{S}$, consisted of the 48 hours SPECT scan of patient A and 3 decayed copies corresponding to 72,96 , and 120 hours following the administration of radioactivity; a uniform effective decay time,
A

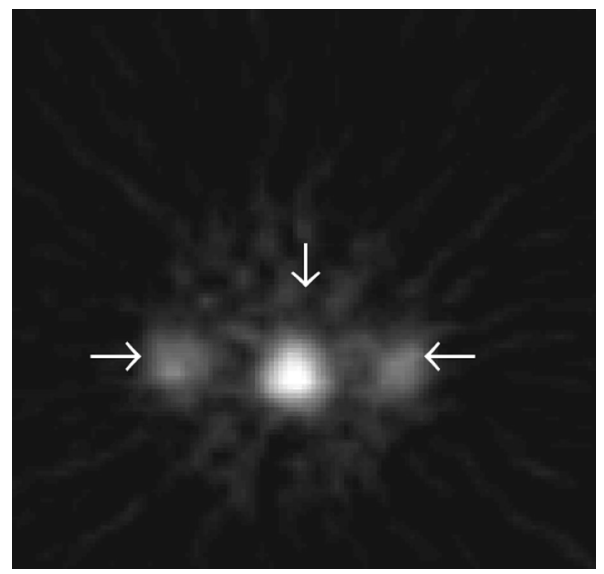

B

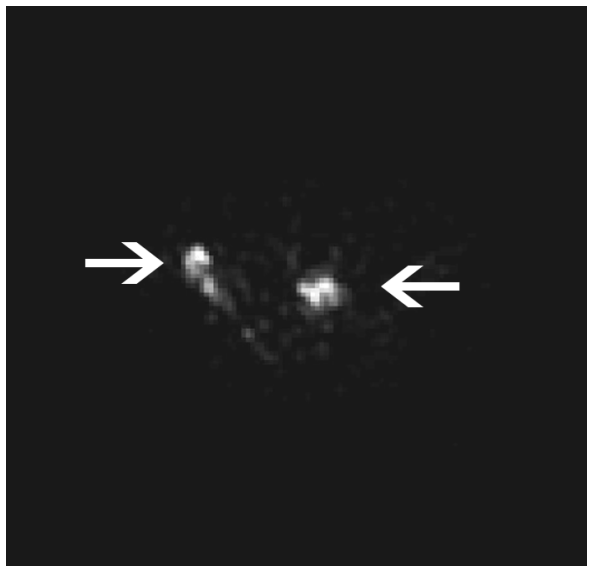

Figure 1. Transaxial slices through patient A ${ }^{131} \mathrm{I}-\mathrm{mIBG}(\mathbf{A})$ and patient $\mathrm{B}{ }^{186} \mathrm{Re}-\mathrm{HEDP}(\mathbf{B})$ single-photon emission computed tomography studies. The tumor sites are right parotid $(\rightarrow)$, odontoid peg $(\downarrow)$, and left parotid $(\leftarrow)$ on $(\mathbf{A})$, and right pelvic bone $(\rightarrow)$ and sacrum $(\leftarrow)$ on $(\mathbf{B})$. 
Table 2. Residual Registration Error (mm) Following Registration with the 3D and 4D Methods and Employing the Correlation Coefficient, Mutual Information, and Sum-of-Absolute Differences Similarity Criteria

\begin{tabular}{lccc}
\hline $\begin{array}{l}\text { Residual registration error } \\
(\mathrm{mm})\end{array}$ & $\begin{array}{c}\text { Correlation coefficient } \\
(\mathrm{mm})\end{array}$ & $\begin{array}{c}\text { Mutual information } \\
(\mathrm{mm})\end{array}$ & $\begin{array}{c}\text { Sum of absolute differences } \\
(\mathrm{mm})\end{array}$ \\
\hline 3D & $3.5 \pm 2.5$ & $3.2 \pm 2.0$ & $7.0 \pm 3.5$ \\
$4 \mathrm{D}$ & $2.4 \pm 1.6$ & $1.9 \pm 1.1$ & $5.3 \pm 2.9$ \\
\hline
\end{tabular}

$T_{\text {eff, }}$, of 20 hours was applied to the entire 3D scan apart from the odontoid peg VOI (Fig. 1A), where a $T_{\text {eff }}$ of 50 hours was employed.

\section{Validation and Comparisons}

The validation process was based on both simulated and patient data. The $\mathrm{A}_{\mathrm{S}}$ simulated data was used to compare the 4D method with sequential $3 \mathrm{D}$ registration. The $\mathrm{A}_{\mathrm{S}}$ dataset was misregistered, and registration was carried out employing both the 4D and sequential 3D methods. The process was repeated for 100 sets of transformations (translation and rotation in 3D) for each similarity criterion, and the residual registration error (i.e., difference between induced and computed transformation) was computed. The 4D method, employing the MI similarity criterion, was also applied to ${ }^{186} \mathrm{Re}$ SPECT studies and the goodness of registration verified by a joint-cursor display tool incorporated in the registration software.

\section{RESULTS}

\section{Simulated Data}

The mean $( \pm \mathrm{SD})$ residual errors employing the $\mathrm{CC}, \mathrm{MI}$ and SAD similarity criteria in sequential 3D and 4D registration methods are summarized in Table 2. In sequential 3D registration, the maximum residual error computed exceeded the voxel size, i.e. $4.7 \mathrm{~mm}$, irrespective of the criterion employed. The incorporation of temporal information resulted in a decrease of the residual error for all three similarity criteria. For the SAD criterion, the maximum residual error was still bigger than the voxel size but was smaller than the corresponding maximum value in the $3 \mathrm{D}$ registration. The similarity criteria rely on the uptake-decay pattern being uniform across the entire volume. The incorporation of temporal information accounts for differences in the uptakedecay pattern and improves the consistency of the similarity criteria as indicated by the decreased residual error. The MI is influenced less by different uptake-decay patterns, compared to the $\mathrm{CC}$
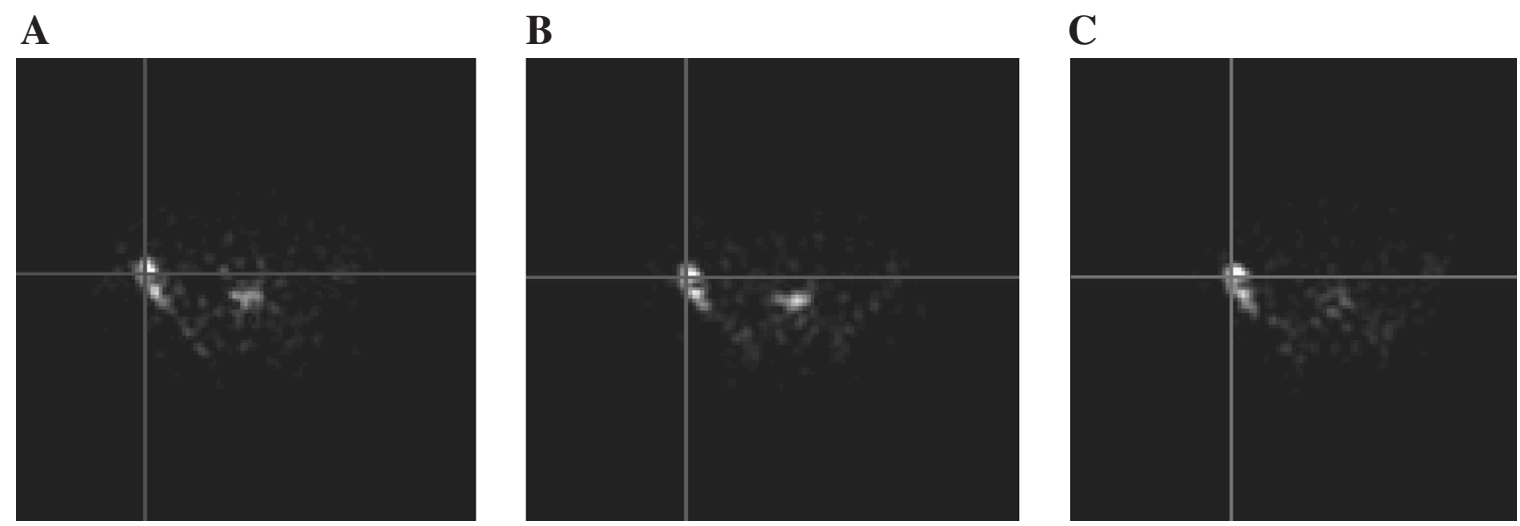

Figure 2. Joint-cursor display of corresponding transaxial slices through the 1-hour (A), the 24 hours $(\mathbf{B})$, and the 72 hours $(\mathbf{C})$ ${ }^{186} \mathrm{Re}-\mathrm{HEDP}$ single-photon emission computed tomography scans following registration using the 4D method and employing the mutual information similarity criterion. 
and SAD criteria, provided that the structure within the volume is not disturbed, explaining why the smallest residual error was computed when the MI criterion was employed, both in the 3D and 4D schemes.

\section{Patient Data}

The 4D method, employing the MI similarity criterion, resulted in accurate registration of the five ${ }^{186} \mathrm{Re}$ HEDP patient studies. In Figure 2, corresponding slices of three SPECT scans $(1,24$, and 48 hours following administration of the radiopharmaceutical) from the patient $B{ }^{186} \mathrm{Re}$ study are shown. Joint cursors over the left pelvic bone confirm that registration employing the 4D scheme was successful.

\section{DISCUSSION}

In this paper, a generalized, iterative 4D registration algorithm for TRT is presented. The algorithm makes the assumption that the spatial distribution within each tumor and/or normal organ VOI remains constant throughout the 4D SPECT sequence. The maximum counts of each tumor site are fitted to a polynomial function, making no assumptions about the underlying uptake-decay pattern. A template 4D dataset is generated based on the polynomial coefficients and the normalization map, and each 3D scan is registered to its corresponding template one.

Voxel-based similarity criteria rely on the uptake-decay pattern being uniform across the volume. The MI criterion does not require for this pattern to be uniform, provided that the structure is not disturbed. That is why the MI criterion results in the smallest residual registration error in sequential registration, especially when different tumor and/or normal sites present with different decay patterns. The incorporation of temporal information accounts for the differences in the uptake-decay patterns, and that is why the performance of all three similarity criteria employed was improved. In a previous work, ${ }^{5}$ the incorporation of temporal information was based on the assumption of a monoexponential decay function describing the tumor TAC; the applicability of the $4 \mathrm{D}$ registration algorithm was thus limited to scans belonging to the decay phase. In this study, polynomial fitting rendered the algorithm independent of the uptake-decay pattern and thus more generalized. However, owing to the limited number of points, usually 5-7 acquired SPECT scans, the choice of the fitting algorithm could be important. The fitting algorithm used assumes equal errors for all data points in a series, an approximation that does not have an effect on the registration process.

The generation of a normalization map is based on the assumption of the spatial distribution within each tumor VOI remaining constant throughout the 4D series. The above assumption does not always hold (e.g., tumors might shrink during therapy), which consists of a limitation for the method's applicability, as it is a limitation for every registration algorithm, based on similarities between the scans to be registered. In the case of the tumor spatial distribution being nonuniform throughout the $4 \mathrm{D}$ series, the definition of subvolumes, within each tumor site, might provide a solution. However, if the change in the tumor geometry over the 4D SPECT series is considerable, accurate registration might not be achieved. Another point, related to the normalization process, is the choice of the reference scan, particularly when early scans are acquired. In this implementation, the first acquired scan was chosen as the reference. However, if there is uptake up to 24 hours following administration, it might be preferable to choose a later scan was the reference. In the ${ }^{186}$ Re-HDEP clinical studies that were analyzed in this paper, the reference was always the first acquired scan i.e., $(\sim 1$ hour following administration), and there was no indication of the algorithm failing to register the SPECT scans.

\section{CONCLUSIONS}

A generalized, iterative, 4D registration algorithm has been developed and validated for TRT dosimetry. The algorithm combines temporal information with 3D similarity criteria to carry out simultaneous registration of a 4D SPECT series. The algorithm was proven more robust, compared to sequential, voxel-based registration methods, because it incorporated the time information, and thus, the registration was tailored to the individual patient study. It also simplified the registration process, as all scans were registered within one session.

\section{REFERENCES}

1. Flux GD, Webb S, Ott RJ, et al. Three-dimensional dosimetry for intralesional radionuclide therapy using mathematical modeling and multimodality imaging. $J$ Nucl Med 1997;38:1059. 
2. Kolbert KS, Sgouros G. Display and manipulation of multimodality images for radiolabeled antibody therapy. Radiology 1998;209(Suppl):41P.

3. Kolbert KS, Hamacher KA, Jurcic JG, et al. 2001, Parametric images of antibody pharmacokinetics in Bi213HuM195 therapy of leukemia. J Nucl Med 2001;42:27.

4. Sgouros G, Squeri S, Ballangrud AM, et al. Patient-specific, 3-dimensional dosimetry in non-Hodgkin's lymphoma patients treated with ${ }^{131} \mathrm{I}$-anti-B1 antibody: Assessment of tumor dose-response. J Nucl Med 2003;44:269.

5. Papavasileiou P, Flux GD, Flower MA, et al. An automated technique for SPECT marker-based image regis- tration in radionuclide therapy. Phys Med Biol 2001; 46:2085.

6. Sgouros G, Kolbert KS, Sheikh A, et al. Patient-specific dosimetry for ${ }^{131}$ I thyroid cancer therapy using ${ }^{124}$ I PET and 3-dimensional-internal dosimetry (3D-ID) software. J Nucl Med 2004;45:1366.

7. Sjögreen K, Ljungberg M, Wingardh K, et al. Registration of emission and transmission whole-body scintillation-camera images. J Nucl Med 2001;42:1563.

8. Chang LT. A method for attenuation correction in radionuclide computed tomography. IEEE Trans Nucl Sci 1978;25:638. 